\title{
Les formes d'affaissement minier dans le bassin ferrifère luxembourgeois
}

Essai de géomorphologie minière

\section{Alex Storoni}

\section{OpenEdition \\ Journals}

Édition électronique

URL : https://journals.openedition.org/rge/3949

DOI : 10.4000/rge.3949

ISSN : 2108-6478

Éditeur

Association des géographes de l'Est

\section{Édition imprimée}

Date de publication : 1 septembre 2001

ISSN : 0035-3213

\section{Référence électronique}

Alex Storoni, «Les formes d'affaissement minier dans le bassin ferrifère luxembourgeois », Revue Géographique de l'Est [En ligne], vol. 41 / 4 | 2001, mis en ligne le 23 juillet 2013, consulté le 28 juin 2022. URL : http://journals.openedition.org/rge/3949 ; DOI : https://doi.org/10.4000/rge.3949

Ce document a été généré automatiquement le 29 septembre 2020

Tous droits réservés 


\section{Les formes d'affaissement minier dans le bassin ferrifère luxembourgeois}

Essai de géomorphologie minière

\section{Alex Storoni}

Thèse soutenue publiquement à l'Université de Nancy 2 le 15 décembre 2000 sous la direction du professeur André Weisrock, 248 pages, glossaire, 6 tableaux, 133 figures, 38 photos, 94 références bibliographiques

\section{Objectifs et méthodes d'étude}

1) L'objectif général de l'étude est de recenser et d'expliquer les nombreuses formes d'affaissement issues de l'exploitation du minerai de fer oolithique sur la partie luxembourgeoise du revers du plateau bajocien (fig. 1). Cet objectif recouvre en fait plusieurs objectifs spécifiques: décrire et expliquer la répartition et la variété des formes d'affaissement en fonction des données du milieu et des techniques d'exploitation minière; expliquer les mécanismes de formation des différentes formes d'affaissement et distinguer les véritables formes d'affaissement des pseudo-formes; enfin dégager une typologie génétique des formes d'affaissement. 
Figure 1 : Croquis morphologique des deux bassins ferrifères luxembourgeois

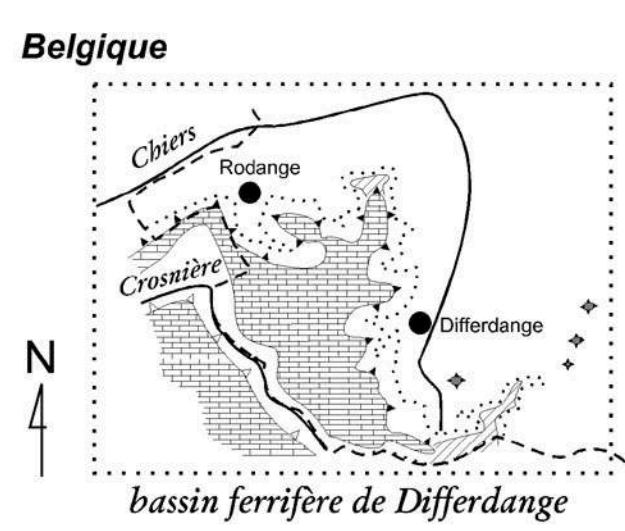

\section{Luxembourg}

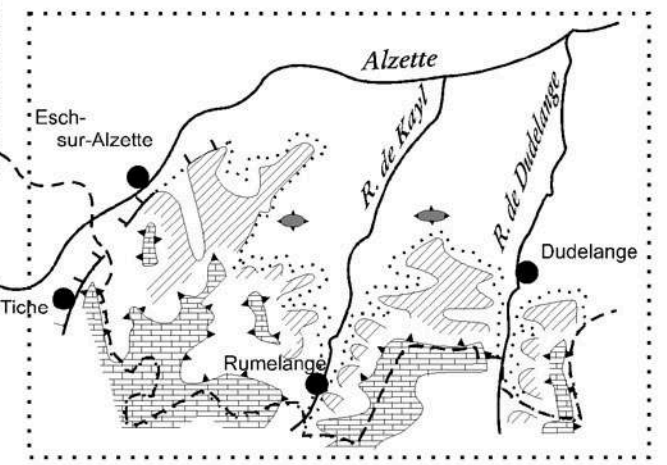

bassin ferrifere d'Esch-sur-Alzette

2 Le problème des risques d'affaissement n'a été abordé que d'une manière indirecte étant donné que les mines de fer ne sont plus accessibles et que de nombreux plans d'exploitation sont introuvables. Un certain nombre d'éléments concernant la nature de ces risques découlent cependant de l'étude de la répartition des creux d'affaissement et de l'observation des effondrements récents.

2) Les méthodes d'étude ont consisté à effectuer sur le terrain, au cours de l'été 1997, un premier inventaire des affaissements du bassin minier. Les principaux affaissements ont été reportés sur des cartes topographiques au 1:10 000. Au printemps 2000, un deuxième inventaire général a été réalisé. Onze cartes de répartition des creux d'affaissement traduisent la situation observée lors de ce deuxième inventaire (fig. 2). 
Figure 2 : La répartition des principales formes d'affaissement au nord du plateau de Differdange : exemple de carte des affaissements

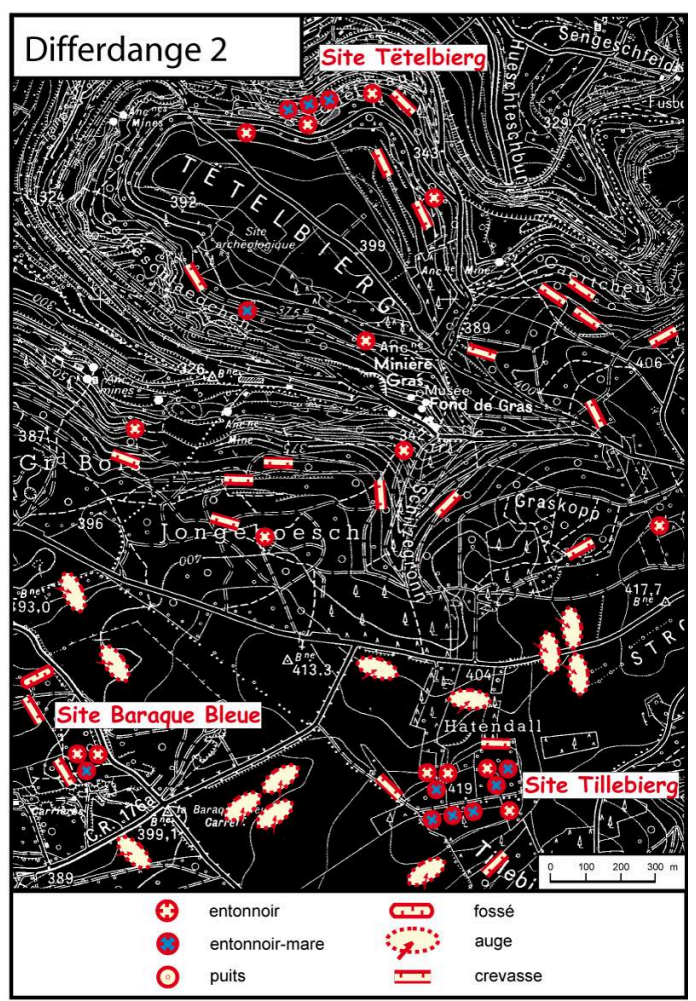

4 Dix sites ont été ensuite retenus en vue d'une étude approfondie (Tableau 1). Leur sélection s'est faite avant tout sur la base de la représentativité des formes d'affaissement qui s'y trouvaient, mais aussi par rapport à la faisabilité de l'étude, qui dépendait très largement de la disponibilité des plans d'exploitation. La morphométrie de détail des creux d'affaissement a été réalisée au cours des étés 1997 à 1999.

Tableau 1 : Les dix sites étudiés en détail et les formes d'affaissement qu'on y rencontre

\begin{tabular}{|l|c|c|c|c|c|c|c|}
\hline \multicolumn{1}{|c|}{ Nom du site } & Puits & Entonnoirs & Mares & Fossés & Auges & Crevasses & $\begin{array}{c}\text { Pseudo- } \\
\text { formes }\end{array}$ \\
\hline Baraque Bleue & & $\mathrm{X}$ & $\mathrm{X}$ & $\mathrm{X}$ & $\mathrm{X}$ & $\mathrm{X}$ & \\
\hline Pellestack & & $\mathrm{X}$ & $\mathrm{X}$ & $\mathrm{X}$ & & $\mathrm{X}$ & \\
\hline Longues Raies & $\mathrm{X}$ & $\mathrm{X}$ & & & & & $\mathrm{X}$ \\
\hline Tillebierg & & $\mathrm{X}$ & $\mathrm{X}$ & & & & $\mathrm{X}$ \\
\hline Origerbësch & & $\mathrm{X}$ & & & & & \\
\hline Koeppchen & & $\mathrm{X}$ & & & $\mathrm{X}$ & \\
\hline Kalebierg & $\mathrm{X}$ & $\mathrm{X}$ & & & & $\mathrm{X}$ & \\
\hline Tëtelbierg & & $\mathrm{X}$ & $\mathrm{X}$ & $\mathrm{X}$ & & $\mathrm{X}$ & $\mathrm{X}$ \\
\hline Grand Bois & & $\mathrm{X}$ & & & & & \\
\hline Eisekaul & & & & & \\
\hline
\end{tabular}

Parallèlement aux études de terrain, des investigations ont été conduites aux Archives de l'Etat, auprès de l'Inspection du Travail et des Mines et auprès de collectionneurs privés en vue de retrouver les plans d'exploitation correspondant aux sites étudiés. 
6 Finalement, la répartition et les formes des creux d'affaissement ont été confrontées aux techniques d'exploitation utilisées et à la géométrie des mines telle qu'elle ressortait des plans d'exploitation.

\section{Les résultats : la typologie des formes}

7 Trois grands ensembles de formes en creux se rencontrent dans le bassin ferrifère luxembourgeois :

1) Les formes d'affaissement directes résultent d'effondrements qui se sont produits dans les mines sous-jacentes et qui n'ont évolué, par la suite, que sous la seule action de l'érosion naturelle.

Les puits aux parois subverticales sont rares. Comme ils sont tous de formation récente et que leur aspect a changé sensiblement au cours des quatre années d'observation, on doit les considérer comme des formes initiales éphémères qui se transforment peu à peu en entonnoirs. Les puits authentiques résultent directement de l'éboulement du toit de la mine et les galeries d'exploitation sont visibles depuis la surface (fig. 3). On rencontre ces puits uniquement aux endroits où les couches de recouvrement sont peu épaisses. L'ouverture rectangulaire des puits-diaclases a été déterminée par le jeu des fissures de la roche sollicitées par les tensions générées à la suite des éboulements au fond. Les puits-entonnoirs ont des formes intermédiaires entre les entonnoirs et les puits. Ils se trouvent uniquement sur les talus.

Figure 3 : Lieu-dit « Eisekaul » près d'Esch-sur-Alzette : puits authentique apparu en avril 1998, à la suite de travaux de terrassement

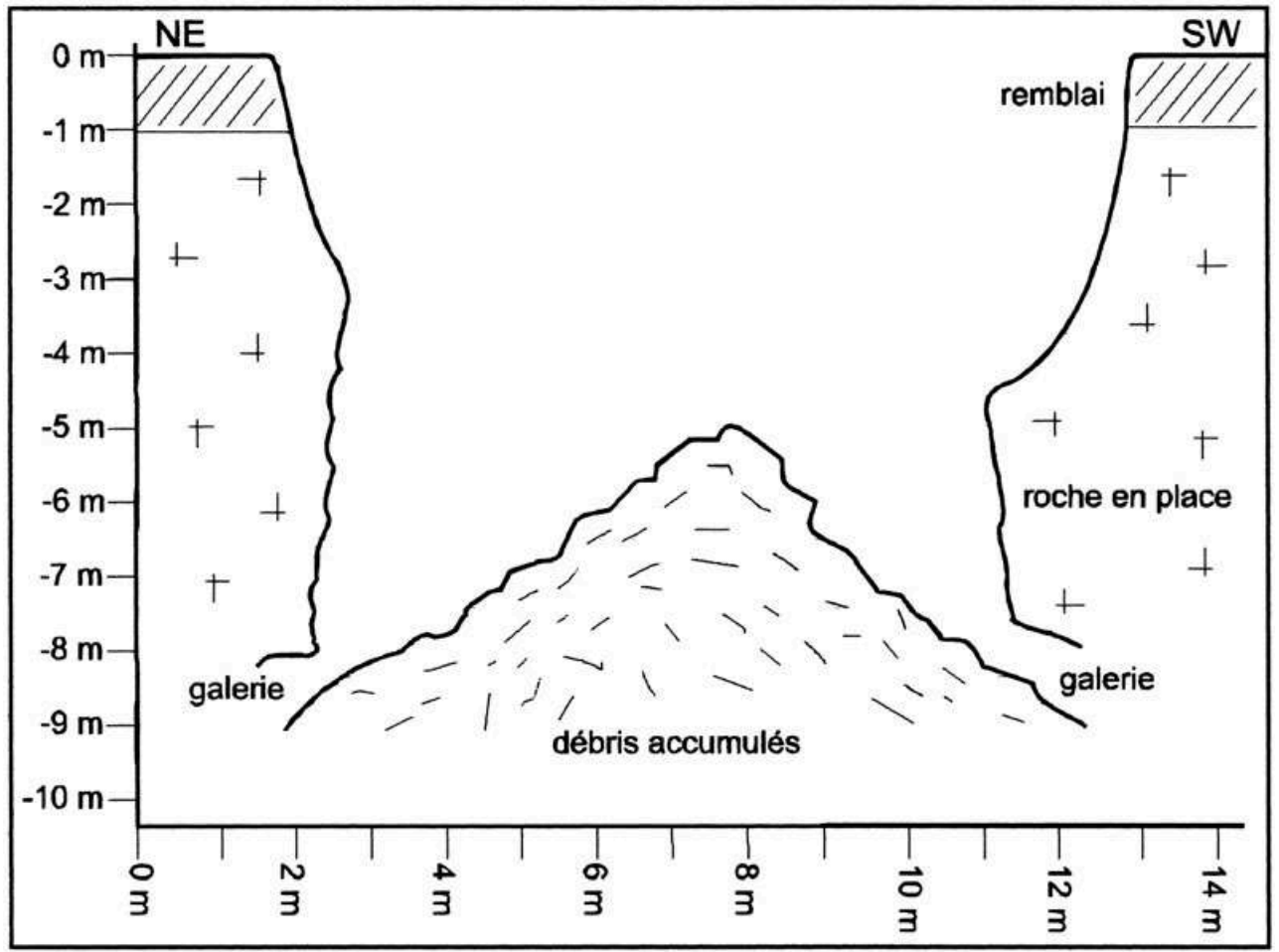

Les formes les plus typiques et les plus courantes sont les entonnoirs qui forment parfois des champs entiers (fig. 5). Les entonnoirs circulaires se rencontrent aux 
endroits où la surface du terrain est plane ou en légère pente. Ils sont en général isolés et constituent des formes pionnières en ce sens que leur proportion diminue au fur et à mesure que le nombre total des creux augmente sur un site donné. Les entonnoirs à plan elliptique se forment, en topographie plane, au-dessus des galeries protégées par des piliers latéraux. Ils ne constituent, dans ce cas, qu'une variante des entonnoirs circulaires. Sur les talus, les entonnoirs ont partout un plan elliptique en même temps qu'un profil dissymétrique. Les entonnoirs coalescents sont formés de plusieurs entonnoirs voisins dont les bords mitoyens se sont recoupés ou qui sont reliés par des tranchées peu profondes. Ils sont fréquents au-dessus des mines à taux de défruitement élevé (fig. 4).

Figure 4 : Lieu-dit « Pellestack » du plateau de Differdange, été 1998 : champ d'entonnoirs coalescents. Le traçage des chantiers de la mine transparaît en surface. II a été établi d'après un plan d'exploitation de la mine Grand Bois de 1926

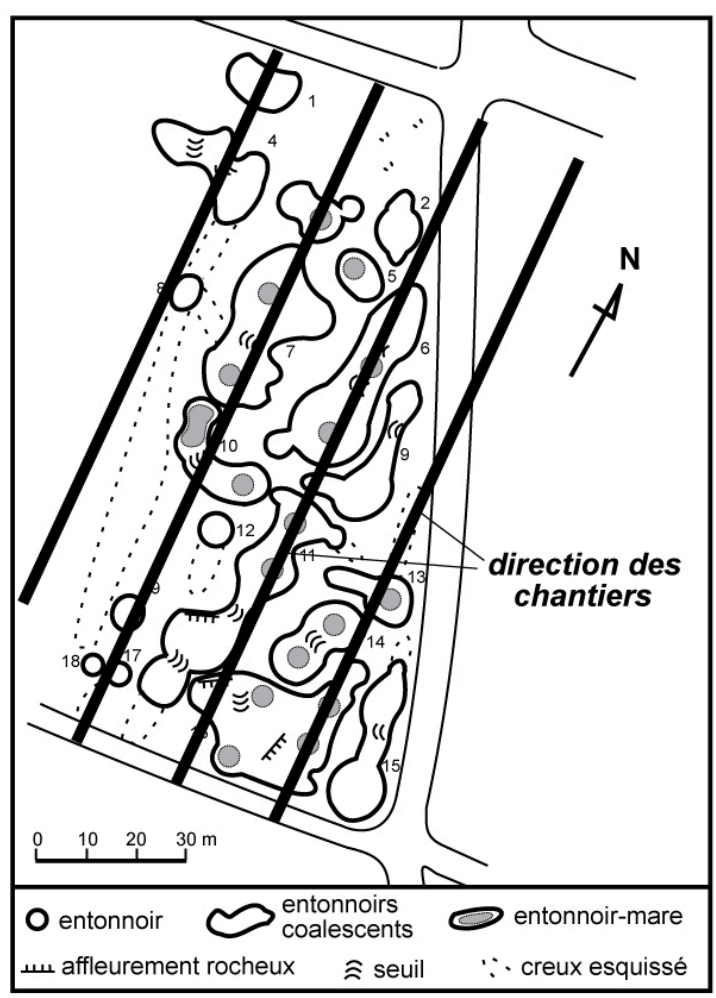


Figure 5 : Champ d'entonnoirs et crevasses sur le plateau de Saulnes-Rodange

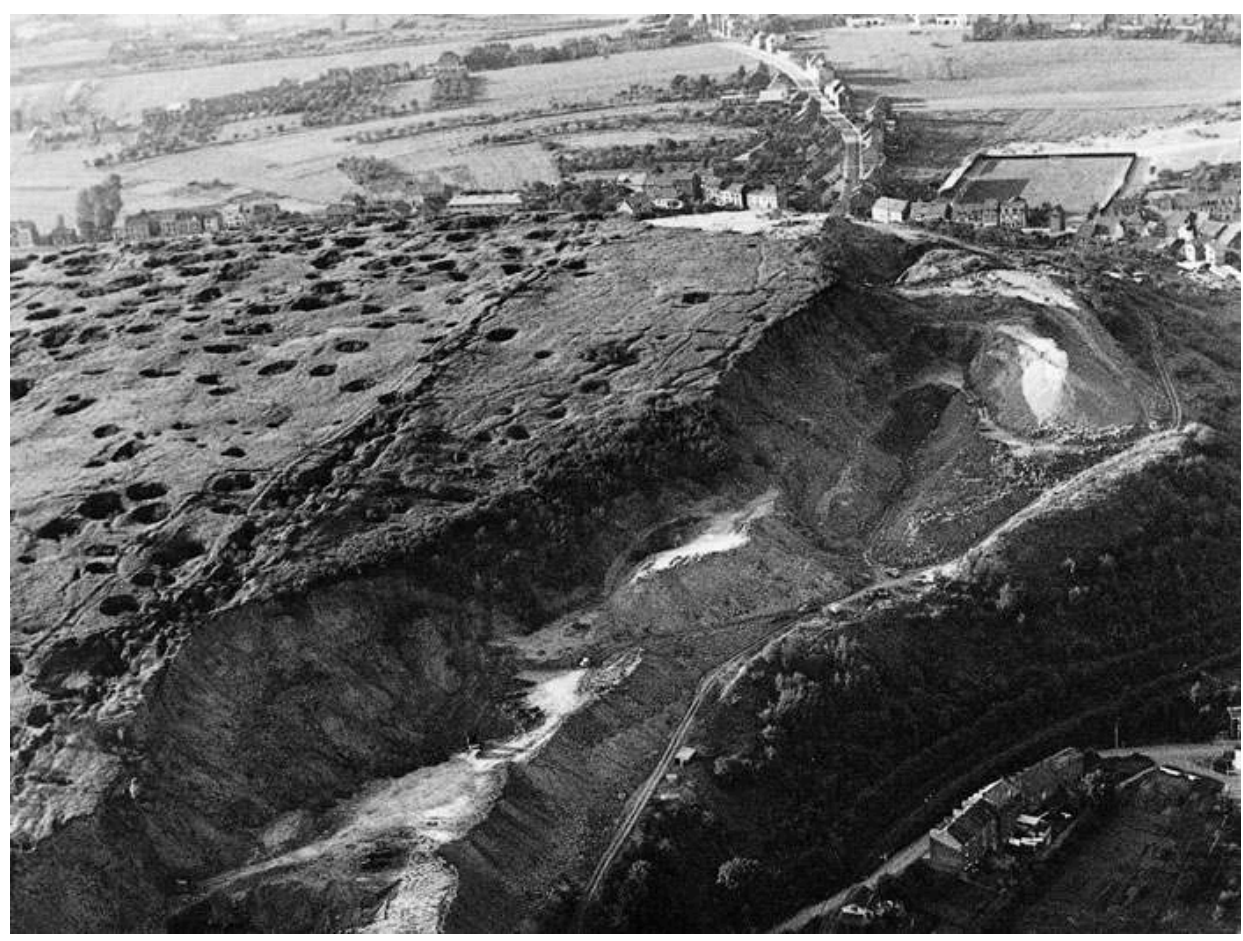

A gauche la France, à droite le Luxembourg. Les crevasses marquent la frontière. Les entonnoirs et les crevasses ont aujourd'hui disparu, à la suite de la réhabilitation des terrains du côté français et de l'extraction des calcaires de couverture du côté luxembourgeois

Copyright: Photothèque de la Ville de Luxembourg, auteur Théo. May, mars 1957

11 Des entonnoirs-mares se trouvent aux endroits du plateau où affleure la formation latéritique du fer fort et sur le replat des marnes micacées qui imperméabilisent les creux d'effondrement au moment où ils se forment.

Les crevasses de plateau (fig. 5) sont, dans tous les cas, associées à des limites de concessions. Elles sont dues aux effets des forces de cisaillement qui s'exercent sur les roches de recouvrement le long des appuis qui subsistent dans les mines. Les crevasses de talus sont plus nombreuses que les crevasses de plateau. Elles sont liées aux forces de traction qui s'exercent sur les roches calcaires de recouvrement très fissurées. Ces crevasses sont souvent associées en faisceaux ramifiés.

13 Les fossés que l'on rencontre çà et là sont en réalité des formes complexes se composant de creux individuels emboîtés. Il en est de même de certaines dépressions irrégulières dont les formes sont parfois difficiles à expliquer parce qu'on n'arrive plus à reconstituer les étapes qui ont conduit au résultat final. Ces dépressions s'observent surtout au-dessus de mines dans lesquelles ont été appliquées successivement différentes techniques d'extraction.

14 2) Les formes d'affaissement dérivées sont également le résultat d'effondrements miniers mais, par la suite, elles ont été fondamentalement altérées par l'homme. Les cuvettes irrégulières et les auges sont restées strictement confinées au domaine agricole. Elles sont le résultat de la réhabilitation des pâturages et des champs qui s'est poursuivie tout au long de l'époque minière. Les auges remplacent aujourd'hui d'anciens champs d'entonnoirs ou des faisceaux de crevasses. Les axes des auges traduisent, en règle générale, la trame des chantiers sous-jacents (fig. 6). 
Figure 6 : Lieu-dit « Baraque Bleue ", été 1999 : une auge (trait continu surimposé à la photo aérienne de 1977) dérivée d'un faisceau de crevasses (traînées blanches de la photo) liées à une limite de concessions (trait discontinu surimposé)

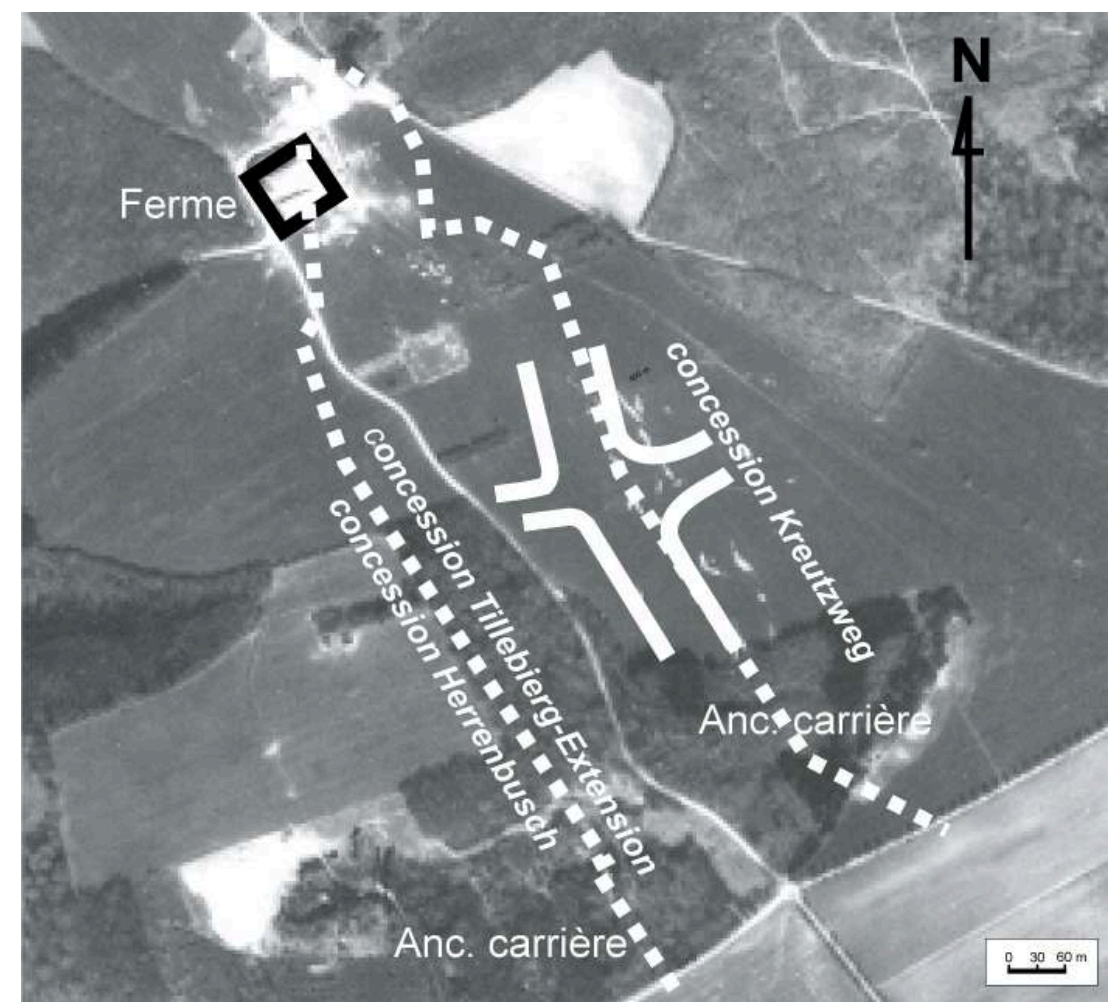

A noter le changement de direction de l'auge qui correspond à celui de la limite de concession

3) Les pseudo-formes ressemblent aux formes d'affaissement authentiques, mais elles ont des origines différentes. Il peut s'agir aussi bien de creux d'origine anthropique (petites carrières, tranchées militaires) que de cavités résultant de chablis. Le type de pseudoformes le mieux représenté est celui des minières de fer fort qui sont souvent - mais pas toujours - accompagnées d'un remblai constitué de l'argile de décalcification rejetée lors du creusement de l'excavation.

\section{Conclusions}

16 1) L'étude des formes d'affaissement du bassin ferrifère luxembourgeois a montré que leur interprétation morphogénétique doit s'accommoder nécessairement des limites imposées par l'ancienneté des mines. A cause de leur inaccessibilité, il est impossible de corréler directement les affaissements en surface avec les éboulements au fond. La corrélation ne peut être faite que par l'intermédiaire des plans d'exploitation dont malheureusement bon nombre ne sont plus disponibles. Toutefois, là où les plans existent, on peut montrer que les affaissements en surface sont toujours en relation étroite avec les travaux d'extraction en profondeur et sont le plus souvent calqués sur la géométrie de la mine. 
17 2) La multiplicité des formes d'affaissement de petite taille qu'on rencontre dans le bassin minier luxembourgeois s'explique :

- par l'étroit compartimentage du relief qui est constitué de deux promontoires de la cuesta bajocienne découpés l'un par les affluents de l'Alzette et l'autre par ceux de la Chiers (fig. 1);

- par la subdivision du sous-sol en deux bassins ferrifères aux caractéristiques divergentes (fig. 1) ;

- par la faiblesse du recouvrement, qui oscille entre quelques mètres et quelques dizaines de mètres au Luxembourg, alors qu'il peut dépasser $200 \mathrm{~m}$ en Lorraine, et par l'exiguïté des concessions luxembourgeoises dont la superficie moyenne n'est que de l'ordre de 35 ha (contre environ 350 ha en Lorraine) ;

- par la présence, aux endroits où la puissance du recouvrement est faible, d'un domaine non concessible partagé entre de multiples petits exploitants ;

- par la variété des modes d'exploitation : exploitation irrégulière des anciennes mines du domaine non concessible, exploitation classique par chambres et piliers, exploitation par piliers perdus, exploitation mixte, à la fois souterraine et à découvert ;

- par le temps inégalement long qui s'est écoulé depuis l'apparition des creux (du milieu du XIXe siècle jusqu'à nos jours) et qui a permis à l'érosion naturelle et anthropique de transformer ces derniers plus ou moins profondément.

18 3) Actuellement, les affaissements ne se produisent plus qu'à un rythme ralenti. Le danger d'une recrudescence des affaissements provient surtout d'une tendance récente à réutiliser, pour les besoins de l'extension de l'infrastructure urbaine, les anciens terrains miniers qui avaient été voués auparavant à l'agriculture ou à la sylviculture. Ce danger est d'autant plus grand que - vu la faiblesse générale du recouvrement - aucun terrain situé au-dessus d'une mine souterraine n'est définitivement à l'abri d'un affaissement.

\section{AUTEUR}

\section{ALEX STORONI}

Centre Universitaire de Luxembourg, 162a, avenue de la Faïencerie, L-1511 Luxembourg 